\title{
Evaluation de la Dynamique Familiale et Position Depressive Familiale: Apport des Méthodes Projectives
}

\author{
Pascal Roman ${ }^{1}$ \\ Université Lumière - Lyon 2
}

\begin{abstract}
RÉSUMÉ - L'auteur propose de mettre au travail, à partir d'une approche groupale-familiale, les conditions dans lesquelles s'établit, au plan des fonctionnements psychiques, la dynamique familiale. A partir de la notion de position dépressive familiale défendue par l'auteur (Roman, 1999), il s'agira d'appréhender la spécificité de l'économie psychique du groupe familiale. Si la méthode projective autorise une approche du fonctionnement psychique de chaque membre de la famille, il apparaît tout à fait pertinent de mettre en perspective les productions projectives au sein de la famille afin de spécifier la spécificité de la configuration de la position dépressive familiale. La présentation d'une situation clinique permet d'illustrer les propositions théoriques et méthodologiques contenues dans cet article.
\end{abstract}

Mots-clés: dynamique familiale; méthodes projectives; transmission psychique; position dépressive familiale; adolescence.

\section{Avaliação da Dinâmica Familiar e Posição Depressiva Familiar: Contribuição de Métodos Projetivos}

\begin{abstract}
RESUMO - O autor propõe trabalhar, a partir de uma aproximação grupal-familiar, as condições sobre as quais se estabelece, no plano de funcionamentos psíquicos, a dinâmica familiar. A partir da noção de posição depressiva familiar defendida pelo autor (Roman, 1999), se tratará de apreender a especificidade da economia psíquica do grupo familiar. Se o método projetivo autoriza uma aproximação do funcionamento psíquico de cada membro da família, torna-se pertinente, então, colocar em perspectiva as produções projetivas no seio da família a fim de especificar a especificidade da configuração da posição depressiva familiar. A apresentação de uma situação clínica permite ilustrar as proposições teóricas e metodológicas contidas neste artigo.
\end{abstract}

Palavras-chaves: dinâmica familiar; métodos projetivos; transmissão psíquica; posição depressiva familiar; adolescente.

\section{The Assessment of the Familial Dynamic and the Depressive Familial Position: Contributions of Projective Methods}

\begin{abstract}
The author suggests to work with a groupal-family approach and with the conditions that the psychical family dynamic are based on. To start with, the family depressive position theory, defended by the author (Roman, 1999), shows groupal-family psychical economy specificities. If the projective method allows a closer view to the family depressive position configuration, thus, it will be important to come up with the projective perspective in the family, in order to specify the specificities of the depressive family configuration. The presentation of a clinical situation illustrates the theoretical and methodological propositions that are shown in this article.
\end{abstract}

Key words: familial dynamic; projective methods; psychical transmission; family depressive position; adolescent.

La notion de parentalité est une notion récente: on en doit la première véritable définition, me semble-t-il, à Houzel (1999) et aux travaux qu'il a menés dans le cadre de situations qui interrogent la qualité du lien familial. Houzel, dans ce contexte, différencie trois aspects de la parentalité: l'exercice de la parentalité (niveau symbolique), l'expérience de la parentalité (niveau de l'investissement subjectif) et la pratique de la parentalité (niveau de l'engagement dans la réalité du lien parental). Ici nous nous intéresserons à ce qui organise la construction de la parentalité, dans le contexte du lien groupal familial, en ce que celui-ci sous-tend la parentalité. Cette

1 Endereço: 5 av P. Mendès, France, 69676, Bron Cedex. E-mail: pascal. roman@univ-lyon2.fr question du groupe familial rencontre, bien sûr, la double question de la filiation (lignée) et de l'alliance, et c'est dans ce double registre que je me propose d'aborder la dynamique groupale familiale, d'une part sur un plan théorique, et d'autre part dans ses implications cliniques et, en particulier, ses implications au plan de la clinique projective.

\section{Clinique du Groupe Familial}

Le mythe familial constitue une notion à partir de laquelle la dimension du groupe familial se trouve en mesure de prendre corps. Selon Kaës (1985), le mythe familial pourrait permettre de contenir et fonder la reconnaissance pour chaque membre du groupe familial de l'existence de chacun: à ce 
titre, il serait le pôle organisateur de l'homéostasie familiale, actualisant les marques singulières du travail de séparation. Le mythe familial consisterait en la forme particulière, individuante, de l'appareil psychique familial en rendant compte tout à la fois d'un pré-investissement de base du lien familial - en tant que signifiant une inscription dans une histoire singulière - et des modalités de l'organisation fantasmatique qui y président. Dans cette perspective, Kaës (1985) propose une définition de la filiation, en lien avec l'élaboration du roman familial chez l'enfant. En effet, toujours selon Kaës, la filiation se construit sur la reconnaissance d'une inscription dans la chaîne des générations: ainsi, la référence commune à un fondement mythique originaire assure une continuité narcissique. Le roman familial, décrit comme structure intermédiaire et transitionnelle au décours de l'établissement de la filiation, servirait alors trois ordres de processus sur lesquels il convient de s'arrêter. Outre le processus de contre-identification au parent du même sexe et de trois traitements de la haine au sein des relations dans le groupe familial, Kaës insiste sur la participation du roman familial au processus: "d'élaboration de la position dépressive dans le moment de séparation des parents, au moment des identifications tierces". Ainsi le roman familial pourrait-il être pensé comme le point de nouage de l'accès à la position dépressive en ce qu'il inscrit celle-ci, dans sa valence essentiellement narcissique, dans les enjeux d'une position dépressive familiale, organisatrice de l'inscription dans la filiation.

Le lien familial doit être entendu, il me semble, comme l'espace au sein duquel se fondent et se déploient les investissements narcissiques et objectaux, au sein du groupe familial - ou de ce qui en tient lieu: le lien familial inscrit, dans la psyché du sujet singulier, les organisateurs inconscients du groupe familial. C'est à partir des travaux des psychanalystes du groupe-Anzieu (1981), et Kaës $(1976,1993)$ en particulier - qu'a pu se développer une pensée du lien familial comme étant inscrit dans des configurations d'interactions débordant le cadre des engagements intersubjectifs entre la mère et son enfant, par ailleurs fort bien décrits par des spécialistes de la psychopathologie de l'enfant tel Lebovici (1983). La notion d'appareil psychique groupal, élaborée par Kaës et Anzieu, puis celle de groupe interne - ou de groupalité interne - défendue par Kaës, proposent en effet un cadre pour se représenter, non seulement les enjeux intersubjectifs des relations entre les appareils psychiques des membres du groupe familial mais encore la manière dont la psyché individuelle est porteuse, dans l'intrapsychique, des investissements propres aux membres du groupe d'appartenance. Ruffiot, A. \& Coll (1990), dans une reprise de ces travaux, propose une définition d'un appareil psychique familial, dont la fonction princeps consisterait à contenir les psychismes individuels et à proposer à la psyché de chacun un méta-cadre de symbolisation des éprouvés individuels. Ruffiot, A. \& Coll se réfère à la capacité de rêverie, décrite par Bion (1962) pour spécifier une fonction onirique de l'appareil psychique groupal: au travers de celle-ci, se trouve potentiellement assurée la circulation des fantasmes du groupe familial, dont les organisateurs concourent à assurer un espace de structuration des fantasmes individuels, tout particulièrement autour de la problématique oedipienne.

La notion d'enveloppe généalogique familiale, mise en évidence et élaborée par Granjon (1987), permet de penser une véritable organisation de l'espace psychique familial qui, comme espace psychique groupal, se fonde sur des alliances in- conscientes (au sens où Kaës a pu le montrer dans ses travaux sur les groupes). Les trois organisateurs principaux de l'enveloppe généalogique familiale apparaissent en premier lieu dans la fonction de délimitation et de frontière (dans le fil de la reconnaissance de la fonction spécifique de contenance, de protection et de structuration identitaire proposée par Anzieu au travers du concept de Moi-peau et des prolongements de celui-ci dans la clinique), en second lieu dans la fonction de différenciation entre les générations, signifiant d'un ordre fondateur de l'interdit de l'inceste et enfin dans celle d'inscription des liens au sein de la famille, inscription soustendue par l'ensemble des dits et des non-dits, des pensés et des impensés de l'histoire familiale, tout particulièrement dans ses enjeux de transmission. Racamier a bien montré, quant à lui, de quelle manière les défauts de structuration du fantasme oedipien au sein du groupe familial mettaient en péril, au travers de ce qui en est transmis au terme des alliances inconscientes, la capacité de chacun de ses membres à investir une place différenciée et stable quant à ses investissement identificatoires. Racamier (1992) décrit cet avatar dans la constitution du lien familial à partir de la figure de l'incestuel, dont il propose qu'elle pourrait constituer un organisateur pathologique au sein du groupe familial: l'incestuel s'appuierait sur la persistance d'un fantasme d'autoengendrement, de déni des origines et, partant, sur un écrasement de l'inscription intergénérationnelle. Dans cette configuration, la parentalité se trouverait elle-même entachée, dans ses différentes composantes, des effets des confusions liées à l'incestuel.

\section{La Position Depressive Familiale}

Pour ma part, je propose de penser ce décentrage de l'intrapsychique à l'intersubjectif permettant de penser la dynamique du lien familial et, à partir de là, de la participation de la parentalité au sein de ce lien, à partir du concept de position dépressive familiale (Roman, 1999), à partir d'une nouvelle lecture du modèle théorique proposé par Klein. Le concept de position dépressive, chez Klein $(1934,1940)$ appartient à un système, inscrit dans le projet d'une modélisation large pour la compréhension des étapes fondatrices de la procédure de séparation-individuation et de l'établissement de la relation objectale. Dans cette mesure, le concept de position dépressive fournit à la clinique un outil précieux pour interroger la qualité des liens intersubjectifs au regard de l'élaboration des procédures de maturation intrapsychique.

Dans la conceptuologie de Klein, la position dépressive apparaît comme une modalité de résolution de l'expérience persécutrice de la rencontre avec l'objet, dans la mesure où celui-ci contiendrait potentiellement la menace d'une rétorsion au regard de l'agressivité sadique-orale développée par l'enfant. C'est ainsi que la position dépressive infantile consisterait en un temps de sortie de la période dépressive, temps de réparation fantasmatique de l'intégrité maternelle, tout à la fois reconnue dans la survivance de celle-ci aux attaques dont elle a été l'objet de la part de l'enfant. La position dépressive infantile mettrait à l'oeuvre une double dynamique, s'établissant, dans une intrication des processus, dans une participation à la constitution du narcissisme de l'enfant d'une part et à son expérience d'une continuité narcissique dans l'ordre des générations d'autre part: ainsi assisterait-on à un double maillage des enjeux de la libido, sur 
les plans narcissique et objectal qui va se traduire en termes d'alliances inconscientes.

Le concept de position dépressive familiale que je propose viserait à rendre compte, au sein du groupe familial, des modalités d'établissement des procédures de séparation/individuation, dans la mesure où ces modalités se trouvent inscrites au sein de ce groupe dans l'ordre des générations. Il me semble que ce concept devrait permettre de mettre au travail, au sein du groupe familial - en tant que l'entité groupale contient les problématiques individuelles - différents niveaux d'investissement de la question identitaire portés par le concept de position dépressive et inhérents à celui-ci: en tant que reprise des expériences précoces de la différenciation sujet/objet telles qu'elles sont portées et élaborées par chacun des membres du groupe familial - en tant que support de réélaboration, dans le champ oedipien, des enjeux propres aux procédures de différenciation inter-sexuelle et inter-générationnelle, mobilisées par les enjeux inter- et trans-générationnels, ainsi que le propose Granjon (1987). La position dépressive familiale pourrait être proposée alors comme une métaphore familiale de la position dépressive infantile décrite par Klein, dans la mesure où elle témoignerait de la capacité du groupe familial à proposer un espace de différenciation suffisant, dans le cadre d'un mouvement de séparation/individuation qui engage les modalités selon lesquelles se sont construites, au sein de la famille, les transactions inconscientes propres à chacune des lignées maternelle et paternelle.

La mise à l'épreuve de la notion de position dépressive familiale dans la pratique des méthodes projectives conduit à identifier un certain nombre d'indicateurs au décours des protocoles de Rorschach et de T.A.T. Au Rorschach, au-delà du contexte expressif du protocole (nombre de réponses, refus, qualité de la verbalisation...), peuvent être regroupés autour de quatre pôles principaux: celui de la discrimination du stimulus, en tant que celle-ci rend compte des modalités d'investissement de l'objet dans sa valence intégrité/morcellement d'une part et dans la perspective de la différenciation figure/fond, Moi/non-Moi, sujet/objet d'autre part... À ce titre, je privilégierai le repérage d'indices tels que la présence de fusions perceptives (contaminations) ou de généralisations abusives (confabulations), de modalités d'appréhension mettant à mal la différenciation forme/fond (avec les réponses de fusionforme - fond en particulier), ou de particularités dans le traitement perceptif du stimulus (réponses dites globales amputées par exemple...) celui du registre du lien à l'objet trouvé, tel qu'il peut s'exprimer sur son versant persécuteur, avec le repérage d'indices de l'attaque de l'objet dans son intégrité: au Rorschach, la marque la plus manifeste tient dans la présence de détails dits oligophrènes, qui signifie une censure dans l'ordre de la représentation - et non pas dans l'ordre du percept visant à porter atteinte à l'objet total celui de la tonalité dépressive du protocole, dans la double dimension de l'affect dépressif, dans le cadre plus large d'une problématique narcissique, dont rendent assez fidèlement compte les réponses intégrant un estompage ou des couleurs achromatiques (Chabert, 1986) et de l'accès à la constitution de l'autre séparé (avec le traitement des différentes figures de double, autour des symétries et des reflets...) - celui de l'élaboration des supports identitaires et identificatoires, comme espace de figuration d'une organisation fantasmatique familiale, avec d'une part l'émergence des réponses humaines (et/ou des formations substitutives) et une attention particulière sur la manière dont elles se présentent à la planche III, d'autre part la présence et la qualité des kinesthésies humaines (et des kinesthésies mineures le cas échéant) dans ce qu'elles engagent du traitement du lien à l'objet (kinesthésie de relation, en référence aux différents registres mis en évidence par Rausch de Traubenberg \& Sanglade, 1984) dans leur élaboration d'une grille de représentation de soi, et enfin le mode de traitement des problématiques paternelle et maternelle, référées respectivement aux planches IV et VII, mais dont d'autres planches peuvent se faire l'écho (planches VI et IX).

$\mathrm{Au}$ T.A.T, ce sont bien sûr les sollicitations de la conflictualité oedipienne qui se présentent de la façon la plus marquante, au regard même de la structure figurative du matériel (Brelet \& Chabert, 2003; Chabert, 1993). Toutefois, le travail sur les protocoles de T.A.T. s'établira à partir de deux axes principaux, que l'on peut relever comme les deux organisateurs de la position dépressive familiale: 1) le premier axe s'intéressera aux modalités selon lesquelles la question de la séparation va pouvoir être élaborée, dans la rencontre avec le matériel, à partir de deux planches qui mobilisent de manière spécifique la dimension de la séparation, les planches $3 \mathrm{BM}$ et $13 \mathrm{~B}$. À partir de là, seront interrogées d'une part la qualité de la prise de position à l'endroit de l'expérience de la séparation dans la mise en jeu de la position dépressive et d'autre part, au travers de la tonalité des récits élaborés, les conditions dans lesquelles l'expérience de la position dépressive va pouvoir constituer un support interne pour la constitution d'un lien à l'objet suffisamment fiable et sécurisant, 2) le second axe concernera de manière spécifique les sollicitations de la conflictualité oedipienne, avec en particulier, la prise en compte de la planche 2 avec le triangle oedipien, la planche 4 pour la confrontation à la figure du couple comme lieu d'ancrage d'une figurabilité de l'origine, les planches 6 et 7 pour ce qu'elles mobilisent la problématique du lien oedipien. Chacune de ces planches est interrogée quant à la reconnaissance de la place de chacun des acteurs, de son inscription dans un lien, et de la qualité de cette inscription, ce qui conduira à spécifier les modalités d'élaboration de lien à l'objet, au travers de configurations expressives qui s'appuient sur la qualité de traitement du stimulus: certains récits présentent le scotome d'une des figures présente sur l'image, dans d'autres, c'est le lien qui fait l'objet d'un scotome, enfin certains s'organisent dans un doute, voire une confusion, quant à une inscription intergénérationnelle.

A partir de là, la clinique m'a conduit à décrire trois grandes figures de la position dépressive familiale, figures éclairées par le matériel clinique issu des méthodes projectives (Rorschach et T.A.T). La figure du clivage, ou schize du lien familial, au sein de laquelle toute question adressée au sujet singulier se trouve atomisée selon deux modalités potentielles: d'une part, dans un retournement sur le sujet de la désorganisation née de la rencontre avec l'objet (vécu de perte de repères, perte de limites) et/ou d'autre part, sans que l'une des modalités ne soit exclusive de l'autre, dans une projection sur l'environnement de la diffraction des objets internes, obérant tout travail de liaison au sens d'une inscription généalogique. Le vécu du lien se trouve ainsi au mieux persécutoire, parfois sans objet. La clinique projective peut 
en rendre compte de manière assez exhaustive, pour chacune des deux épreuves: perceptions et représentations morcelées, faisant l'objet de distorsions, référence à des logiques de violence, implicites ou explicites qui signent la mise en danger du sujet dans sa rencontre avec l'objet, banalisations outrancières... La figure de la confusion, ou forme de l'agrégat familial qui s'organise à partir d'un déni de l'altérité, déni qui vise à protéger l'enveloppe familiale, en ce qu'elle contient et organise l'ensemble des enveloppes psychiques individuelles, des effets schizoïdes et/ou paranoïdes de la rencontre avec l'objet. On assiste alors à un rabattement des investissements narcissiques et objectaux selon un modèle que l'on peut rapprocher du modèle de la séduction: la rencontre avec la figure de l'inceste y est omniprésente, dans la mesure de la difficulté des adolescents à faire jouer affects et représentations dans la rencontre d'excitations qui, tant internes qu'externes, traumatisent des réseaux de sens fortement fragilisés. Les collusions représentatives, autour des représentations humaines en particulier, la surchauffe de l'excitation face à des sollicitations qui laminent le pare-excitation, les ratés de l'organisation oedipienne au travers de scotomes et confusions, constituent autant d'indices qui permettent le repérage des troubles de l'inscription transgénérationnelle, et, partant, les empêchements quant à l'élaboration de la position dépressive familiale. C'est dans ce contexte que j'ai proposé le modèle du co-étayage indifférencié pour qualifier ce mode de relation à l'objet. La figure de l'idéal familial, espace potentiel de l'élaboration de la position dépressive familiale dans laquelle on peut reconnaître une potentialité de mise au travail de la position dépressive familiale, au décours du jeu engagé, à partir du matériel comme des représentations qu'il va faire naître, dans la mobilisation de ressources étayées sur des objets internes suffisamment construits dans leur participation familiale, dans l'élaboration de scénarios propres à permettre une articulation des motions subjectivantes et objectivantes... la possibilité de faire vivre, au travers de la réponse au Rorschach ou du récit au T.A.T., l'altérité de l'objet, témoigne de l'ouverture de la position dépressive familiale comme support d'une circulation fantasmatique, et un dégagement du lien dépressif familial.

\section{Clinique du Lien Familial, ou la Parentalité en Question}

On comprend alors que chacune de ces figures porte une mobilisation particulière de la parentalité au sein du groupe familial, dans la mesure où la parentalité, on l'a vu, se construit en appui sur la dynamique groupale familiale, dynamique de l' appariement, au sein de l'appareil psychique familial des appareils psychiques individuels.

L'enjeu de ces propositions, tout à la fois théorique et clinique, est double: d'une part, ces propositions permettent d'envisager une lecture "familiale" de protocoles d'épreuves projectives lors de passations individuelles, d'autre part, elles autorisent de nouvelles perspectives dans une analyse de protocoles recueillis "en famille", selon la méthodologie présentée par Orgiazzi (1993, 1994).

Je proposerai d'apporter une brève illustration clinique pour montrer de quelle manière une lecture des enjeux groupaux-familiaux quant à la construction de la parentalité peut permettre d'éclairer la compréhension, tout à la fois individuelle et familiale, de la souffrance psychique, du point de vue des troubles de la parentalité. Cette illustration clinique est issue d'une situation de consultation familiale (père, mère, adolescent), au décours de laquelle chacun va se trouver, à tour de rôle, en situation de dénoncer la folie de l'autre. M. et Mme X. consultent pour leur fils âgé d'un peu plus de 17 ans (on pourrait dire plus justement "autour de leur fils"), fils que l'on appellera Vincent, et qui est présenté comme le symptôme au sein de la famille. Cette désignation à la place du symptôme s'inscrit dans une configuration où domine un conflit de couple important depuis de nombreuses années. La demande des parents vise prioritairement la souffrance de leur fils, souffrance que ce dernier dénie, considérant que cette souffrance n'existe que dans la pathologie de sa mère.

La mère, quant à elle, considère que c'est le lien entre le père, présenté comme tout à la fois fantasque, rigide et absent qui est problématique. Ce dernier, plutôt en retrait, pense que le lien entre Vincent et sa mère est pathologique. C'est dans ce contexte qu'une passation familiale d'épreuves projectives (Rorschach et T.A.T) leur est proposée afin de tenter d'une part d'ouvrir un questionnement dans un contexte où les choses se présentent de manière particulièrement nouées et d'autre part de bénéficier d'un support (que l'on peut aussi entendre dans le sens d'une médiation) pour aborder, d'un point de vue psychopathologique, ce qui apparaît comme une souffrance massive dans les liens au sein du groupe familial.

A l'issue des entretiens et passations individuelles qui fondent ce dispositif de passation de projectifs "en famille", je propose les éléments de lecture qui suivent: il semble que la place des chacun des trois membres de la famille soit régie par des liens qui se jouent dans le registre de ce que l'on pourrait appeler une séparation impossible, dans la mesure où la séparation porte en elle un risque qui apparaît difficile à assumer pour chacun, au regard des fragilités narcissiques respectives de chacun (père, mère, fils); en filigrane, l'expression de cette problématique dans le registre du mode de lien au sein du couple parental, constitue une des figures de la précarité des investissements du lien, la dimension de la triangulation oedipienne semble se trouver en difficulté dans sa potentialité structurante des liens à l'intérieur de la famille, que ce soit sur le plan du repérage de la place différenciée de chacun des acteurs familiaux ou de la qualité de leur prise de position dans les liens intersubjectifs; ainsi, tout autant que l'insécurité repérable dans les fonctions de soutien et de maintenance (avec des effets de maîtrise de la position de l'autre qui fait figure d'empiètement) est sensible, la fonction organisatrice du tiers apparaît comme effacée, les positions d'évitement du conflit, qui apparaissent comme modalité quelque peu partagée dans la dynamique des liens à l'intérieur de la famille, tendent à obturer la possible conflictualisation des positions, au profit d'émergences que l'on peut qualifier de violentes, qu'elles se traduisent ou non en acte. Afin d'apporter des éléments de discussion clinique de la notion de position dépressive familiale et de sa contribution à la compréhension de la dynamique du lien familial, je propose d'envisager particulièrement les réponses produites à la planche III au Rorschach, dont on connaît la sollicitation tout à la fois identitaire et identificatoire, et les récits proposés à la planche 2 au T.A.T, à l'égard de laquelle la construction de la triangulation oedipienne (croisement de la différence des sexes et des générations) se trouve mobilisée. Les productions à ces deux planches permettent d'illustrer les expressions projectives d'une impossible séparation, d'une position dépressive familiale marquée par la figure de la confusion, et ouvrant sur une modalité de lien dépressif, 
en abordant la dimension transfantasmatique au sein de la famille (qui peut aussi prendre la forme d'un dispositif de passation en couple), prévoit la passation d'épreuves individuellement pour chacun des membres d'une famille, sans communication entre eux entre les temps de passation d'une même épreuve. Il est ensuite proposé à chacun un temps de restitution de la compréhension de leur fonctionnement psychique individuel, puis en famille, un temps de présentation de ce qui transparaît des enjeux trans-fantasmatiques (transdéfensifs et trans-aménagements) qui peuvent de dégager d'une lecture conjointe de ces protocoles et des pistes de travail qui en émergent.

\section{Rorschach, planche III}

La présentation de la production de chacun (père, mère et Vincent) sera suivi d'un bref commentaire clinique, dont les données seront rassemblées en forme de mise en perspective des trois protocoles et abordées spécifiquement, du point de vue de Vincent.

Monsieur X.: "il y a toujours une symétrie d'ailleurs depuis le début... ben la tête d'un individu avec deux femmes au-dessus". Enquête: "deux yeux (D7), ou deux formes féminines ou deux oiseaux et puis aussi on dirait le noeud-papillon au-dessus".

On note une identification humaine problématique dans son caractère unitaire, la première réponse se référant uniquement à une partie d'humain; par ailleurs, l'identification, en un second temps, des représentations féminines, marquées par leur localisation, reprise à l'enquête ("au dessus") ne permet pas d'évoquer un lien entre les personnages. On notera que ces représentations tendront à se détériorer à l'enquête pour devenir des "formes".

Madame X.: “des personnages au restaurant... une fête... avec des lampions... c'est pour ça que j'ai pensé restaurant... des gens qui se saluent de manière un peu protocolaire... noeudpapillon et puis courbettes". Enquête: "des gens qui se saluaient ou qui étaient à table”.

La construction des représentations humaines est proposée sans mention de l'appartenance sexuée; le lien énoncé est pris dans la contrainte ("protocolaire"), puis comme dévitalisé et limitée à sa forme figurée ("courbettes"), dans un rabattement sur la dimension perceptive.

Vincent: “alors deux guitares de chaque côté en rouge euh... j'ai l'impression de voir deux coeurs aussi, voir aussi deux danseuses, deux femmes comme ça avec le postérieur vers l'arrière un peu courbée vers l'avant, ou bien un papillon aussi au milieu papillon rouge, là au milieu on dirait une sorte de table ou de tambour sur lequel il y a les danseuses qui jouent comme ça avec les jambes écartées, en perspective, sinon ça fait penser un peu aux zébrures d'un zèbre au niveau des taches qui se truquent... (D7) V. là ça fait un peu un espèce d'insecte avec les yeux, les dents pointues et les mandibules de chaque côté... une espèce d'araignée ou de mante religieuse ou de piranha même... avec deux gros yeux et puis un noeudpapillon aussi et puis deux taches de sang".

La construction de représentations humaines suit une saisie des parties rouges, interprétées pour parties à partir de leur parti- cipation colorée ("coeur", "papillon rouge"), témoignant d'une ouverture à la dimension sensorielle de la planche. Les représentations humaines, féminines, ouvrent l'évocation d'une scène contrastée: en mouvement avec les "danseuses"), elle se trouve fortement défendue par des références au perceptif tendant à figer les représentations humaines ("postérieur vers l'arrière", "courbée vers l'avant", "jambes écartées, en perspective"). Par ailleurs, on peut noter que ces représentations féminines se trouvent assez rapidement associées à une menace, liée au féminin, au travers du renversement de la planche ("insecte aux dents pointues", "mante religieuse", "piranha"). Au total, on peut mesurer de quelle manière la construction des représentations humaines se trouve dominée par la référence à la posture, signifiant une forme de gel pulsionnel. Le lien se trouve comme immobilisé, figé dans le perceptif, y compris dans la référence à une identité sexuée qui se présente, d'une manière ou d'une autre, comme fragile.

L'absence de dynamique d'échange entre les personnages confère un caractère de fragilité des supports pour la construction du lien. Du point de vue de Vincent, on peut penser que la menace associée à la représentation humaine dans sa définition (insistance sur la posture) et dans sa dynamique relationnelle (redoublement narcissique de la représentation, sans jeu identificatoire possible) traduit une construction interne au sein de laquelle il serait maintenu en emprise mortifère: la saisie au plan perceptif permet de contrôler l'émergence pulsionnelle... et les potentiels de liaison qu'elle contient.

\section{T.A.T, planche 2}

La présentation des productions à cette planche se déroulera selon la même organisation que précédemment.

\begin{abstract}
Monsieur X.: “ouh là c'est carrément champètre... ou alors c'est le... là c'est un, c'est une histoire je dirais conflictuelle entre l'homme qui travaille dur, qui fait ses sillons et sa femme peut-être à côté qui est plus livresque, qui n'est pas très liée, qui n'est pas très liée, qui s'est dit... y a un fossé dans les sillons entre le travail de force de la nature et un côté un peu intellectuel... et puis un troisième personnage qui est peut-être la mère, la garante un peu de l'ordre, un peu au-dessus, qui voudrait que tout soit comme d'habitude parce qu'il y a un schisme... qui pourrait être le fille d'ailleurs, l'homme, la femme et la fille... l'histoire, c'est un conflit entre le travail de la terre et le travail des méninges".
\end{abstract}

Le récit proposé par Monsieur X. est marqué par une difficulté dans la conceptualisation du lien homme/femme; on peut noter à cet égard les effets de répétition et les troubles dans le déroulement du récit (associations courtes en particulier). Le doute sur les places de chacun des personnages de la scène, et l'impossibilité d'une élaboration dans un registre oedipien ouvre sur un déplacement des représentations sur la scène du social, au travers d'une intellectualisation ("l'histoire, c'est un conflit entre le travail de la terre et le travail des méninges").

Madame X.: "photo d'époque... l'agriculture d'aujourd'hui... y a beaucoup de choses... une jeune fille studieuse qui va avec ses livres à l'Université, qui pense à l'avenir, à ses responsabilités... à côté il y a l'agriculteur, un homme robuste, solide qui laboure, au jour le jour... dans un paysage qui est très beau, peut-être la mer au fond... et là une femme... est-ce qu'elle attend un enfant? 
elle est fatiguée... elle s'appuie contre un tronc d'arbre, elle se repose... elle fait le point, plus âgée que la jeune fille, marquée par les maternités... moment de répit, elle prend le soleil".

Le récit ne comporte pas de liens entre les personnages. L'évocation de la relation parentale se limite à la référence à une maternité présumée et à un rabattement sur la définition du lien selon des critères que l'on peut qualifier de narcissiques ("plus âgée que la jeune fille").

Vincent: (rit en voyant la planche): "là déjà c'est une jeune écolière qui sort des cours qui habite à la campagne en Italie qui sort des cours et qui passe dans la nature ça doit être au mois... des labours ou des semences... au printemps ou à l'automne, qui passe par les champs pour rentrer chez elle et elle rentre peut-être à cause du beau paysan à côté du cheval, c'est pour ça qu'elle fait tous les jours le détour par laferme voisine avant de rentrer chez elle bosser ses cours...".

Face à cette planche, le récit proposé s'appuie sur un scotome du personnage féminin en position maternelle, et ouvre sur l'évocation d'un lien de séduction entre la jeune fille et le personnage masculin. La mise en perspective des récits proposés par les deux parents et Vincent ouvre sur la reconnaissance de la place des effets de clivage comme mode d'évitement de la conflictualité oedipienne; dans ce contexte, le lien oedipien ne peut trouver à se construire sur des images stables et conflictualisables. Du point de vue de Vincent, on peut penser que le traitement discrètement érotisé, de la planche 2 du T.A.T, dans un registre de séduction, témoigne de l'incertitude de la construction des imagos parentales élaborées dans le lien à ses parents.

\section{Position Depressive Familiale: une Figure de la Confusion?}

Sans doute peut-on considérer que la difficile résolution de la position dépressive familiale dont témoigne cette clinique familiale, et qui prend ici la forme d'une figure de la confusion, avec l'émergence d'éléments laissant à penser un mode de fonctionnement psychique s'organisant sur le mode du clivage, traduit un trouble de la parentalité. Se donnerait à voir une forme pathologique de la construction de la parentalité, marquée, en particulier, par un gel de l'inscription générationnelle. La fragilité dans la constitution des identités sexuées qui émerge des protocoles des parents de Vincent, et la souffrance dont les marques du clivage organisent la trace quant à l'engagement dans le lien (tout à la fois lien narcissique et lien objectal) traduisent la difficulté d'une mise en jeu de la place de Vincent dans une fantasmatique des origines: en effet, la dynamique familiale semble s'être construite sur un déni du lien d'alliance comme fondateur de l'origine, laissant ouverte une figure de la filiation impossible, au sens où elle ne se trouverait pas inscrite dans une conflictualisation et un ordonnancement des places au sein de la famille.

Dans cette configuration, la position dépressive familiale se trouve empêchée dans sa dimension structurante des liens au sein du groupe familial, la qualité de ceux-ci se trouvant rabattue sur des modalités binaires du type dépendance/exclusion, chaque membre de la famille, dans une forme d'indifférenciation des générations, se trouvant alternativement convoqué à chacune de ces modalités, dans des formations de "couple": dépendance père et mère dans l'exclusion du fils, dépendance mère-fils dans l'exclusion du père, dépendance père-fils dans l'exclusion de la mère. La thématique de la folie tend alors à circuler, paradoxalement au regard de son énoncé, au sein du groupe familial comme le reste d'une tentative différenciatrice et de lutte contre la confusion.

L'analyse de la nature de la construction de la parentalité telle qu'elle émerge de la qualité du lien familial, en appui sur le concept de position dépressive familiale mis à l'épreuve des méthodes projectives, ouvre des perspectives tout à la fois au plan de l'évaluation des souffrances du lien familial qu' au plan de l'élaboration des dispositifs de prise en charge.

\section{References}

Anzieu, D. (1981). Le groupe et l'inconscient. Paris: Dunod.

Bion, W. R. (1962). Aux sources de l'expérience. Paris: P.U.F.

Brelet, F. \& Chabert, C. (2003). Nouveau Manuel du T.A.T. Paris: Dunod.

Chabert, C. (1986). Narcissisme au Rorschach. Bulletin de la Société Française du Rorschach et des Méthodes Projectives, 33, 15-40.

Chabert, C. (1993). Narcissisme et relations d'objet à l'adolescence: apport des épreuves projectives. Bulletin de la Société Française du Rorschach et des Méthodes Projectives, 37, 183-194.

Granjon, E. (1987). L'enveloppe généalogique familiale, in Actes du C.O.R, Arles, p. 73-75.

Houzel, D. (1999). Les enjeux de la parentlité. Toulouse: Erès.

Kaës, R. (1976). L'appareil psychique groupal - Constructions du groupe. Paris: Dunod.

Kaës, R. (1985). "Filiation et affiliation, quelques aspects de la réelaboration du roman familial dans les familles adoptives, les groupes et les instituitions", Gruppo, 1, p. 23-46.

Kaës, R. (1993). Le groupe et le sujet du groupe. Paris: Dunod.

Klein, M. (1934). Contribution à l'étude de la psychogenèse des états maniacodépressifs. in Klein, M., Essais de Psychanalyse, Paris, Payot, 1976, p. 311-340.

Klein, M. (1940). Le deuil et ses rapports avec les états maniaco-dépressif. in Klein M., Essais de Psychanalyse, Payot, 1976 341-369.

Lebovici, S. (1983). Le nourrisson, la mère et le psychanalyste. Paris: Bayard.

Orgiazzi, I. (1993). Rorschach et fonctionnement familial dans un cas d'anorexie mentale. Bulletin de la Société Française du Rorschach et des Méthodes Projectives, 37, 103-123.

Orgiazzi, I. (1994). Le T.A.T. de l'univers familial. Bulletin de Psychologie, XLVII (416), 426-431.

Racamier, P.-C. (1992). Le génie des origines. Paris: Payot.

Rausch de Traubenberg, N. \& Sanglade, A. (1984). Représentation de soi et relation d'objet au test de Rorschach: grille de représentation de soi. Revue de Psychologie Appliquée, 34(1), 41-57.

Roman, P. (1999). La position dépressive familiale: un modèle pour penser la séparation - Apport des méthodes projectives. Psychiatrie de l'Enfant, XLII(1), 129-172.

Ruffiot, A. \& Coll. (1990). La thérapie familiale psychanalytique. Paris: Dunod.

Recebido em 08.10.2007

Primeira decisão editorial em 01.11.2007

Versão final em 03.11.2007

Aceito em 10.01.2008 